03,18

\title{
Рекомбинация в бесщелевой квантовой яме в гетероструктуре $\mathrm{HgTe} / \mathrm{CdHgTe}$
}

\author{
() В.Я. Алешкин ${ }^{1}$, А.А. Дубинов ${ }^{1}$, В.И. Гавриленко ${ }^{1}$, С.Г. Павлов ${ }^{2}$, H.-W. Hübers ${ }^{2,3}$ \\ ${ }^{1}$ Институт ффизики микроструктур РАН, \\ Нижний Новгород, Россия \\ ${ }^{2}$ Institute of Optical Sensor Systems, German Aerospace Center (DLR), \\ Berlin, Germany \\ ${ }^{3}$ Institut für Physik, Humboldt-Universitat zu Berlin, \\ Berlin, Germany \\ E-mail: aleshkin@ipmras.ru
}

Поступила в Редакцию 26 октября 2021 г.

В окончательной редакции 26 октября 2021 г.

Принята к публикации 28 октября 2021 г.

\begin{abstract}
Рассмотрены три механизма рекомбинации неравновесных носителей в бесщелевой нелегированной квантовой яме в гетероструктуре $\mathrm{HgTe} / \mathrm{CdHgTe}$. Вычислены зависимости средней по ансамблю вероятности рекомбинации (обратного времени рекомбинации) от концентрации неравновесных носителей для рекомбинации с испусканием оптических фононов, рекомбинации с испусканием двумерных плазмонов и излучательной рекомбинации.
\end{abstract}

Ключевые слова: бесщелевая квантовая яма $\mathrm{HgTe}$, механизмы рекомбинации неравновесных носителей.

DOI: 10.21883/FTT.2022.02.51947.227

\section{1. Введение}

Бесщелевые квантовые ямы (с нулевой шириной запрещенной зоны) могут быть использованы для создания детекторов, смесителей и генерации излучения среднего и дальнего инфракрасного диапазонов. Высказывалось много предложений использовать для этих целей графен (см., например, [1]). Однако, несмотря на успехи в демонстрации усиления [2,3] и детектирования (см., например, [4-6]) терагерцового излучения в структурах на основе графена, эти работы не привели к созданию коммерческих приборов. По-видимому, это связано с отсутствием в настоящее время технологии получения высококачественного графена, пригодного для создания таких устройств. Однако бесщелевые квантовые ямы можно создавать и на основе гетероструктур $\mathrm{HgTe} / \mathrm{CdHgTe} \mathrm{[7],} \mathrm{технологии} \mathrm{получения} \mathrm{которых} \mathrm{хоро-}$ шо отработаны [8]. Важной для работы, как детекторов и смесителей, так и источников излучения является такая характеристика материала как время жизни неравновесных носителей. Для графена вопрос о временах жизни неоднократно исследовался как экспериментально (см., например, работу [9] и ссылки в ней), так и теоретически [10-13]. Напротив, для бесщелевых квантовых ям $\mathrm{HgTe} / \mathrm{CdHgTe}$ к настоящему времени эта характеристика остается практически неизученной.

Цель настоящей работы состоит в теоретическом изучении механизмов рекомбинации и вычислении времен жизни неравновесных носителей в бесщелевой квантовой яме $\mathrm{HgTe} / \mathrm{Cd}_{0.7} \mathrm{Hg}_{0.3} \mathrm{Te}$. Рассмотрены три механизма рекомбинации: рекомбинация на оптических фононах в HgTe, рекомбинация на плазмон-фононных модах и излучательная рекомбинация.

\section{2. Электронный спектр бесщелевой структуры}

Рассмотрим квантовую яму $\mathrm{HgTe}$ толщиной $6.2 \mathrm{~nm}$, окруженную барьерами $\mathrm{Cd}_{0.7} \mathrm{Hg}_{0.3}$ Те. Будем полагать, что она выращена на плоскости (013), поскольку эта плоскость предпочтительна для роста методом молекулярно-лучевой эпитаксии [8]. На рис. 1 представлен спектр электронов в этой квантовой яме, рассчитанный с использованием четырехзонной модели Кейна с учетом деформационных эффектов. Детали расчета можно найти в работе [14]. Температура решетки полагалась равной $4.2 \mathrm{~K}$. Для простоты в расчетах не были учтены спиновые расщепления электронных подзон из-за понижения симметрии на гетерогранице и отсутствия центра симметрии [15], что практически не влияет на темпы рассматриваемых механизмов рекомбинации.

Из рис. 1 видно, что при такой толщине квантовой ямы рассматриваемая структура действительно является бесщелевой, а вторая электронная зона Е2 располагается на $0.321 \mathrm{eV}$ выше первой подзоны E1 в точке $k=0$. Поскольку наибольший интерес представляет рассмотрение рекомбинации неравновесных носителей, возбуждаемых квантами излучения дальнего и длинноволновой части среднего инфракрасного диапазонов с существенно меньшими энергиями, в дальнейшем мы ограничимся учетом процессов рекомбинации электронов из подзоны 


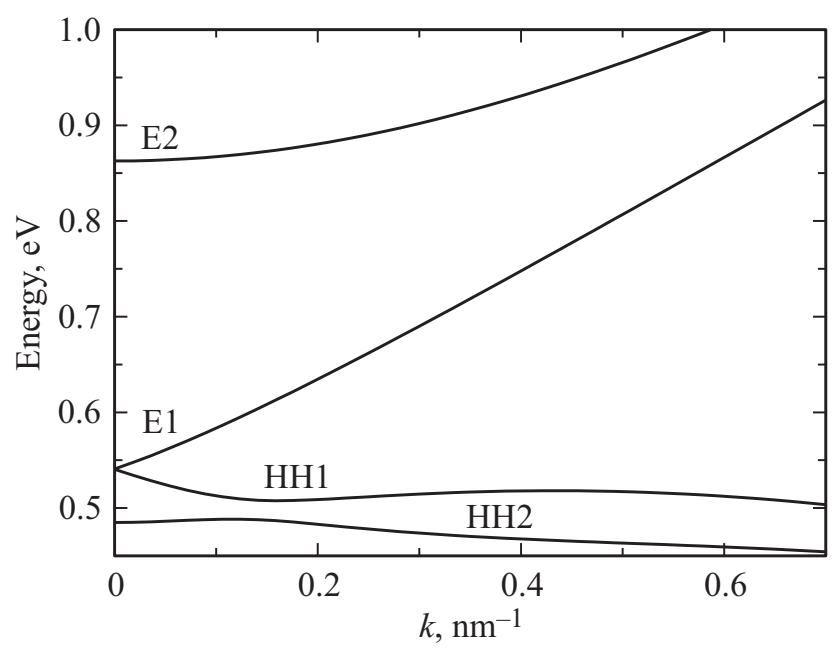

Рис. 1. Спектр электронов в квантовой яме $\mathrm{HgTe} / \mathrm{Cd}_{0.7} \mathrm{Hg}_{0.3} \mathrm{Te}(013)$ толщиной $6.2 \mathrm{~nm}$.

E1 и дырок из подзоны НН1 (вероятность переходов в нижележащие дырочные подзоны существенно ниже).

\section{3. Рекомбинация с участием объемных оптических фононов в $\mathrm{HgTe}$}

Хорошо известно, (см., например, работу [16]) что оптические фононы в квантовых ямах можно разделить на две группы: объемоподобные (bulklike) и поверхностные. В рассматриваемом случае поверхностные фононы гибридизуются с колебаниями электронной плотности в квантовой яме - двумерными плазмонами. Рекомбинация с участием таких гибридных мод рассмотрена в следующем разделе. В объемоподобных модах электрический потенциал продольных фононов имеет вид

$$
\begin{aligned}
\varphi(\boldsymbol{\rho}, z)= & \left(a_{q} \exp (i \mathbf{q r})+a_{q}^{*} \exp (-i \mathbf{q r})\right) \theta\left(d_{Q W} / 2-|z|\right) \\
& \times \begin{cases}\cos \left(\pi n z / d_{Q W}\right), & n=1,3 \ldots \\
\sin \left(\pi n z / d_{Q W}\right), & n=2,4 \ldots\end{cases}
\end{aligned}
$$

где $\mathbf{q}$ - волновой вектор фонона в плоскости квантовой ямы, $d_{Q W}$ - ширина квантовой ямы (яма занимает область $\left.|z|<d_{Q W} / 2\right), a_{q}$ - характеризует величину потенциала, $\theta(z)$ - функция Хевисайда. Выполнив процедуру вторичного квантования поля оптических фононов (см. Приложение), можно найти следующее выражение для оператора электрического потенциала

$$
\begin{aligned}
\varphi(\boldsymbol{\rho}, z)= & -A\left(c_{\mathbf{q}} \exp (i \mathbf{q r})+c_{\mathbf{q}}^{+} \exp (-i \mathbf{q r})\right) \theta\left(\frac{d_{Q W}}{2}-|z|\right) \\
& \times \begin{cases}\cos \left(\pi n z / d_{Q W}\right), & n=1,3 \ldots \\
\sin \left(\pi n z / d_{Q W}\right), & n=2,4 \ldots\end{cases}
\end{aligned}
$$

где $c_{\mathbf{q}}^{+}, c_{\mathbf{q}}-$ операторы рождения и уничтожения оптического фонона,

$$
A=\sqrt{\frac{4 \pi \hbar \omega_{L}}{\bar{\kappa} S d_{Q W}\left(q^{2}+\left(\frac{\pi n}{d_{Q W}}\right)^{2}\right)}},
$$

$\omega_{L}$ - циклическая частота продольного оптического фонона, $S-$ площадь квантовой ямы, $\bar{\kappa}^{-1}=\kappa_{\infty}^{-1}-\kappa_{0}^{-1}$, $\kappa_{\infty}$ и $\kappa_{0}-$ высокочастотная и низкочастотная диэлектрические проницаемости материала квантовой ямы.

Электронные состояния в квантовой яме полностью определяются заданием волнового вектора $\mathbf{k}$ и индекса $s$, который включает номер подзоны и спиновое состояние. Поэтому волновую функцию электрона можно представить в виде

$$
\Psi_{s, \mathbf{k}}(\mathbf{r})=S^{-1 / 2} \exp (i \mathbf{k r}) \psi_{s, \mathbf{k}}(z)
$$

Используя (2) можно найти следующее выражение для вероятности рекомбинации дырки с волновым вектором $-\mathbf{k}$ и индексом $s^{\prime}$ (электрон переходит из $\mathbf{k}+\mathbf{q}, s^{\prime}$ в состояние $\mathbf{k}, s)$ :

$$
\begin{aligned}
w_{s, s^{\prime}}(\mathbf{k})= & \frac{2 \omega_{L} e^{2}}{\bar{\kappa} d_{Q W}} \int d^{2} q \delta\left(\varepsilon_{s^{\prime}}(\mathbf{k}+\mathbf{q})-\varepsilon_{s}(\mathbf{k})-\hbar \omega_{L}\right) \\
& \times f_{s^{\prime}}(\mathbf{k}+\mathbf{q}) \sum_{n} \frac{\left|g_{s^{\prime}, s}(\mathbf{q}, \mathbf{k}, n)\right|^{2}}{\left(q^{2}+\left(\frac{\pi n}{d_{Q W}}\right)^{2}\right)}
\end{aligned}
$$

где $-e-$ заряд электрона, $\varepsilon_{s}(\mathbf{k})-$ энергия электрона в $s$-ом состоянии с волновым вектором $\mathbf{k}, f_{s}(\mathbf{k})-$ функция распределения электронов,

$$
\begin{aligned}
g_{s^{\prime}, s}(\mathbf{q}, \mathbf{k}, n)= & \int_{-d_{Q W} / 2}^{d_{Q W} / 2} d z \psi_{s^{\prime}, \mathbf{k}+\mathbf{q}}^{+}(z) \psi_{s, \mathbf{k}}(z) \\
& \times \begin{cases}\cos \left(\pi n z / d_{Q W}\right), & n=1,3 \ldots \\
\sin \left(\pi n z / d_{Q W}\right), & n=2,4 \ldots\end{cases}
\end{aligned}
$$

Полный темп рекомбинации можно записать в виде

$$
R=\sum_{s, s^{\prime}} \frac{1}{(2 \pi)^{2}} \int d^{2} k w_{s^{\prime}, s}(\mathbf{k})\left[1-f_{s}(\mathbf{k})\right],
$$

где индекс $s$ пробегает состояния зоны проводимости, а индекс $s^{\prime}$ - валентной зоны. Отметим, что (6) описывает полный темп рекомбинации. Наблюдаемый темп рекомбинации равен разности полного темпа рекомбинации и темпа тепловой генерации носителей. Мы будем рассматривать случай, когда концентрация неравновесных носителей много больше концентрации равновесных носителей. В этом случае тепловой генерацией можно пренебречь и можно ввести среднюю по ансамблю вероятность рекомбинации носителей $W=R / n$, где $n=p-$ концентрация неравновесных носителей. 


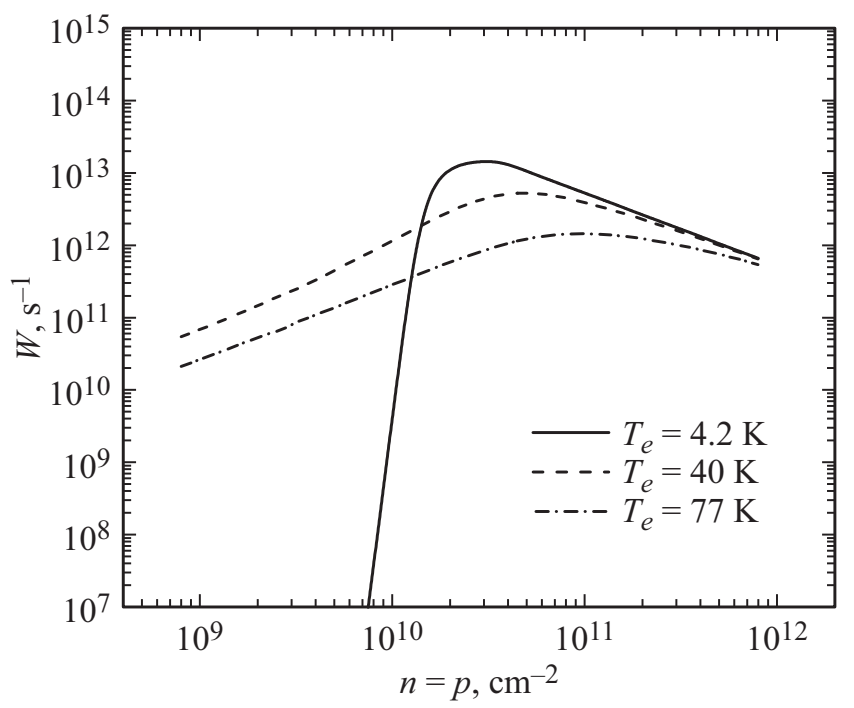

Рис. 2. Зависимость средней по ансамблю вероятности рекомбинации дырки на объемоподобном фононе от концентрации носителей для трех эффективных температур неравновесных носителей.

На рис. 2 представлены результаты расчетов $W$ от концентрации носителей. В расчетах концентрации дырок и электронов полагались одинаковыми, функции распределения электронов и дырок описывались распределением Ферми-Дирака с эффективной электронной температурой $T_{e}$, которая могла принимать три значения 4.2, 40 и $77 \mathrm{~K}$. На рисунке хорошо видно наличие максимума в зависимости $W$ от концентрации, который убывает и смещается в область больших концентраций с ростом эффективной температуры неравновесных носителей. Убывание $W$ при превышении концентрации, соответствующей максимуму $W$, обусловлено невозможностью участия в рекомбинации электронов и дырок, обладающих кинетической энергией, превышающей энергию продольного оптического фонона. При превышении концентрации, соответствующей максимуму, доля таких носителей увеличивается и поэтому $W$ убывает. Уменьшение $W$ с падением концентрации носителей в области, где концентрация меньше соответствующей максимуму $W$, связано с уменьшением числа пар электрондырка, участвующих в рекомбинации, когда разность энергий Ферми электронов и дырок становится меньше энергии оптического фонона. Отметим, что для пары электрон-дырка, кинетическая энергия которой меньше энергии оптического фонона, рассматриваемый процесс рекомбинации запрещен законом сохранения энергии.

\section{4. Рекомбинация с участием плазмон-фононной моды}

Неравновесные носители в бесщелевых квантовых ямах $\mathrm{HgTe}$ могут рекомбинировать с испусканием двумерных плазмонов. В работе [17] была изучена рекомби- нация с участием двумерных плазмонов в узкощелевых квантовых ямах, однако не был рассмотрен случай бесщелевой квантовой ямы.

Взаимодействие двумерных плазмонов с оптическими фононами барьерных слоев (полагается, что ширина квантовой ямы много меньше длины волны плазмона) приводят к образованию высокочастотной и низкочастотной плазмон-фононных мод, аналогично тому, как это происходит в объемном полупроводнике [18]. Частота высокочастотной моды превышает частоту продольного оптического фонона в барьере, а частота низкочастотной моды меньше частоты поперечного оптического фонона в барьере. Такие плазмон-фононные моды являются поверхностными оптическими колебаниями решетки, сосредоточенными около границ квантовой ямы.

На рис. 3 приведены зависимости энергии плазмонфононных возбуждений от волнового вектора при различных концентрациях носителей. Для нахождения зависимости частоты плазмон-фононных мод от волнового вектора была использована методика, подробно изложенная в работе [19]. Точки „окончания“ законов дисперсии плазмон-фононных мод обусловлены „включением“ ${ }^{6}$ затухания Ландау, которое делает эти моды плохо определенными из-за больших потерь. В условиях затухания Ландау мнимая часть частоты плазмона становится порядка действительной части, и время затухания таких плазмонов становится много меньше характерного времени рекомбинации. Поэтому в условиях затухания Ландау вклад плазмонов в рекомбинацию несущественен. По этой причине учитывался вклад в рекомбинацию только плазмонов, для которых отсутствует затухание Ландау.

При расчетах эффективная температура электронного и дырочного газов принимала три значения: 4.2, 40 и $77 \mathrm{~K}$. Отметим, что повышение эффективной температуры носителей заряда слабо сказывается на законе

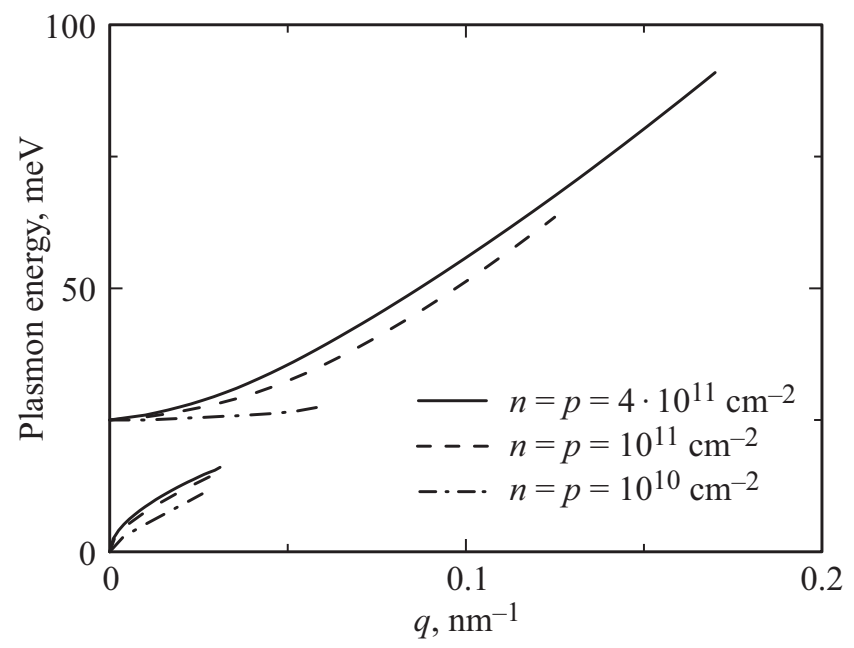

Рис. 3. Зависимости энергий кванта плазмон-фононных мод от волнового вектора для трех концентраций неравновесных носителей. $T_{e}=4.2 \mathrm{~K}$. 
дисперсии рассматриваемых плазмонов [19], однако оно уменьшает величину волнового вектора, при котором становится существенным затухание Ландау.

Электрическое поле плазмона имеет две компоненты: одна из них направлена вдоль волнового вектора, а другая - по нормали к квантовой яме (по оси $z$ ). Та компонента, которая направлена вдоль волнового вектора, является четной функцией от $z$, если начало отсчета выбрать в середине квантовой ямы. Поскольку характерный масштаб ее изменения в $z$ направлении $1 / q$, а ширина квантовой ямы полагается много меньшей $1 / q$, то эту компоненту можно считать постоянной внутри квантовой ямы. Другая компонента электрического поля является нечетной функцией $z$. На границах квантовой ямы эти компоненты примерно равны по величине. Поэтому вероятность перехода электрона из зоны проводимости в валентную зону квантовой ямы под действием электрического поля плазмона выше для компоненты электрического поля, лежащей в плоскости квантовой ямы. Для нахождения вероятности таких переходов можно использовать оператор компоненты вектор-потенциала, направленной вдоль волнового вектора, приведенный в работе [17]:

$$
\begin{aligned}
\mathbf{A}_{\|} & =-c \sum_{\mathbf{q}, j} \frac{\mathbf{q}}{\omega} \sqrt{\frac{2 \hbar}{S q^{2}}\left(\frac{\partial \chi(\mathbf{q}, \omega)}{\partial \omega}\right)^{-1}} \\
& \times\left(d_{\mathbf{q}, j} \exp (i \mathbf{q} \mathbf{r}-i \omega t)+d_{\mathbf{q}, j}^{+} \exp (-i \mathbf{q} \mathbf{r}+i \omega t)\right),
\end{aligned}
$$

где $\chi(\mathbf{q}, \omega)$ - поляризуемость электронно-дырочного двумерного газа (выражение для вычисления $\chi(\mathbf{q}, \omega)$ можно найти в работе [17]), $d_{\mathbf{q}}^{+}$и $d_{\mathbf{q}}$ - операторы рождения и уничтожения кванта плазмон-фононной моды, индекс $j$ введен для обозначения высокочастотной и низкочастотной моды. Используя (7) можно получить следующее выражение для темпа рекомбинации с испусканием плазмонов

$$
\begin{aligned}
& R=\frac{2}{(2 \pi)^{3}} \sum_{j, s, s^{\prime}} \int d^{2} k d^{2} q \frac{\mid \mathbf{v}_{s, \mathbf{k} ; s^{\prime}, \mathbf{k}+\left.\mathbf{q} q\right|^{2}}}{q^{2} \omega_{j}^{2}(\mathbf{q})}\left(\frac{\partial \chi\left(\mathbf{q}, \omega_{j}\right)}{\partial \omega_{j}}\right)^{-1} \\
& \times \delta\left(\varepsilon_{s}(\mathbf{k})-\varepsilon_{s^{\prime}}(\mathbf{k}+\mathbf{q})-\hbar \omega_{j}(\mathbf{q})\right)\left(1-f_{s^{\prime}}(\mathbf{k}+\mathbf{q})\right) f_{s}(\mathbf{k}),
\end{aligned}
$$

где $\mathbf{v}_{s, \mathbf{k} ; s^{\prime}, \mathbf{k}+\mathbf{q}}$ - матричный элемент оператора скорости, индекс $s$ пробегает состояния зоны проводимости, а индекс $s^{\prime}$ - валентной зоны.

На рис. 4 приведены зависимости средней по ансамблю вероятности рекомбинации с испусканием квантов плазмон-фононных мод $W=R / n$. Сплошные линии соответствует суммарной рекомбинации включающей испускание квантов как высокочастотной так и низкочастотной мод. Штриховые линии соответствует испусканию только квантов низкочастотной моды. Из рисунка видно, что основную роль в рассматриваемом механизме рекомбинации играют процессы с испусканием квантов высокочастотной моды. Исключением из этого

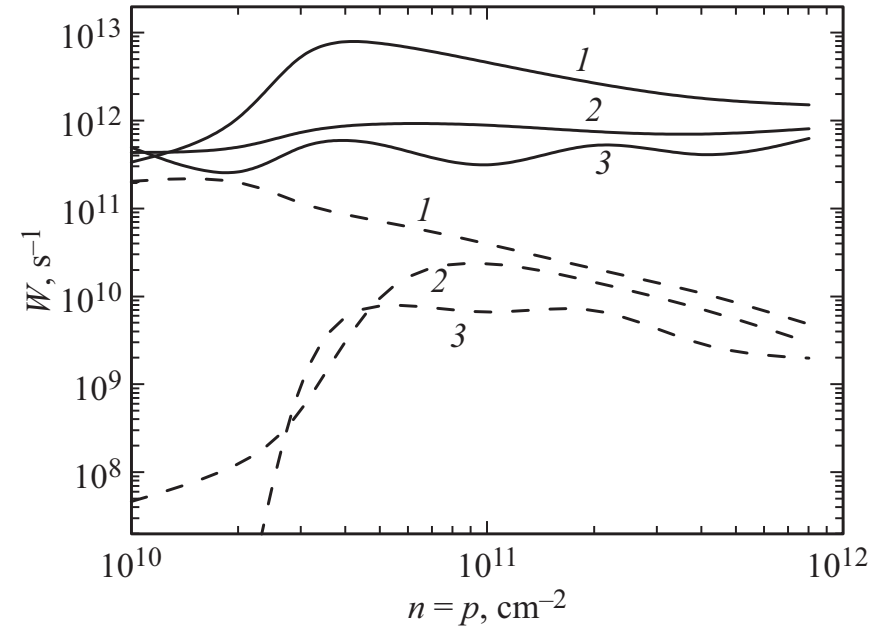

Рис. 4. Зависимости вероятности рекомбинации с испусканием кванта плазмон-фононной моды от концентрации неравновесных носителей (сплошные линии). Пунктирные линии соответствуют вероятности рекомбинации с испусканием кванта низкочастотной плазмон-фононной моды. Цифры 1, 2, 3 соответствуют $T_{e}=4.2 \mathrm{~K}, T_{e}=40 \mathrm{~K}$ и $T_{e}=77 \mathrm{~K}$.

утверждения является случай, когда $T_{e}=4.2 \mathrm{~K}$ и концентрация неравновесных носителей меньше $2 \cdot 10^{10} \mathrm{~cm}^{-2}$. Из рисунка хорошо видно, что вероятность рекомбинации является немонотонной функцией от концентрации неравновесных носителей и что ее величина уменьшается с ростом эффективной температуры носителей.

\section{5. Излучательная рекомбинация}

Этот механизм наиболее медленный среди рассматриваемых механизмов рекомбинации, и его рассмотрение здесь имеет в основном сравнительно-иллюстративную цель. Отметим, что излучательная рекомбинация в твердом растворе $\mathrm{Cd}_{x} \mathrm{Hg}_{1-x}$ Те изучалась в работе [20].

Вероятность спонтанного перехода электрона, обладающего волновым вектором $\mathbf{k}$, с испусканием фотона из состояния зоны проводимости $s$ в незанятое электроном состояние валентной зоны $s^{\prime}$ равна

$$
w_{s^{\prime}, s}(\mathbf{k})=\frac{4 \sqrt{\kappa} e^{2} \omega}{3 \hbar c^{3}}\left|\mathbf{v}_{s^{\prime}, s}(\mathbf{k})\right|^{2},
$$

где $\mathbf{v}_{s^{\prime}, s}(\mathbf{k})=\mathbf{v}_{s, \mathbf{k} ; s^{\prime}, \mathbf{k}}$. Величина, обратная излучательному времени рекомбинации (частота излучательной рекомбинации), равна отношению темпа излучательной рекомбинации к концентрации неосновных носителей. Для полупроводника $n$-типа или в случае, когда $n=p$ ее можно записать в виде [21]:

$$
W=\frac{\sum_{s, s^{\prime}} \int d^{2} k w_{s, s^{\prime}}(\mathbf{k}) f_{s}(\mathbf{k})\left(1-f_{s^{\prime}}(\mathbf{k})\right)}{\sum_{s} \int d^{2} k f_{s}(\mathbf{k})} .
$$




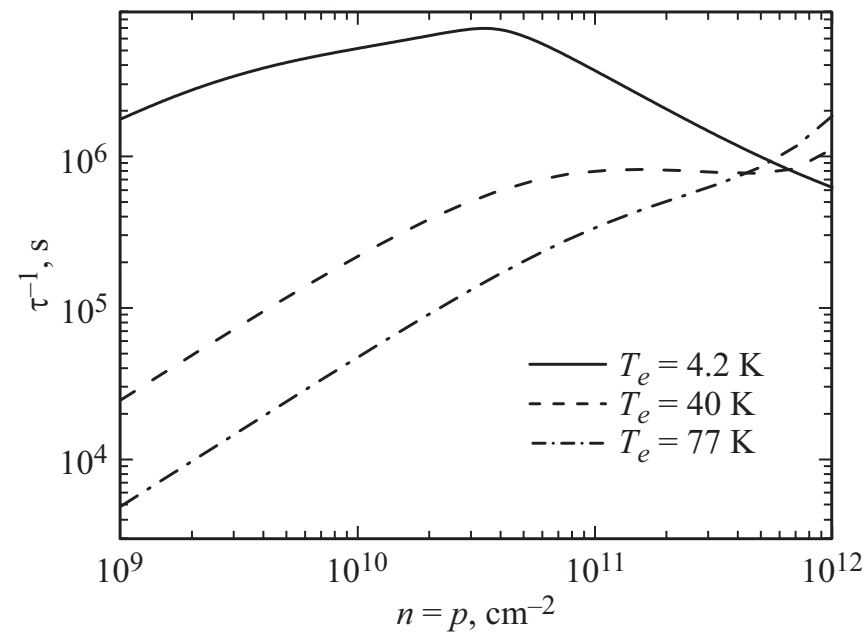

Рис. 5. Зависимость $W(n)$ для излучатенльной рекомбинации для трех эффективных температур.

На рис. 5 приведены вычисленные зависимости $w(n)$ для трех эффективных температур неравновесных носителей.

Из рисунка видно наличие максимума на зависимости $W(n)$ при $T_{e}=4.2 \mathrm{~K}$, который обусловлен заполнением боковых экстремумов дырками (см. рис. 1) с ростом концентрации неравновесных носителей. Дырки в боковых экстремумах не принимают участия в излучательной рекомбинации, поскольку отсутствуют электроны в зоне проводимости с волновыми векторами, соответствующие боковым экстремумам. Из сравнения рис. 2, 4 и 5 видно, что излучательная рекомбинация является самой медленной из рассматриваемых механизмов рекомбинации.

\section{6. Заключение}

В заключении приведем основные результаты работы. Вычислены зависимости от концентрации неравновесных носителей вероятностей рекомбинации в бесщелевой квантовой яме $\mathrm{HgTe}$ для трех механизмов: рекомбинации с испусканием оптических фононов, рекомбинации с испусканием двумерных плазмонов и излучательной рекомбинации. Показано, что наиболее эффективными механизмами рекомбинации среди рассмотренных являются рекомбинация с испусканием оптических фононов и двумерных плазмонов. Максимальные вероятности рекомбинации для этих двух механизмов имеют порядок $10^{13} \mathrm{~s}^{-1}$. Такие короткие времена безызлучательных механизмов рекомбинации неравновесных носителей указывают на неперспективность использования бесщелевых квантовых ям для генерации фотонов, и, возможно, плазмонов. Для этих целей более перспективными выглядят квантовые ямы, ширина запрещенной зоны которых превышает энергию оптического фонона.

\section{Приложение}

Квантование оптических бононов в квантовой яме

Энергию продольных колебаний решетки можно записать в виде

$$
\begin{aligned}
& \sum_{j} \bar{M} \mathbf{r}_{j}^{2} / 2+\bar{M} \omega_{L}^{2} \mathbf{r}_{j}^{2} / 2 \\
& =\frac{\bar{M}}{2 a^{3}} \int d z \int d x d y\left(\mathbf{v r}^{2}(x, y, z)+\omega_{L}^{2} \mathbf{r}^{2}(x, y, z)\right),
\end{aligned}
$$

где $\mathbf{r}_{j}-$ относительное смещение атомов в $j$-ой элементарной ячейке, $\bar{M}-$ приведенная масса элементарной ячейки, $a^{3}-$ объем элементарной ячейки. Поскольку длина волны рассматриваемых оптических фононов много больше постоянной решетки, то вместо $\mathbf{r}_{j}$ можно ввести функцию $\mathbf{r}(x, y, z)$, которую можно рассматривать как функцию непрерывного аргумента. В продольных колебаниях вектор смещения следующим образом связан с величиной электрического поля, действующего на ион [18]:

$$
\mathbf{r}=\frac{e^{*} \mathbf{E}}{\bar{M}\left(\omega_{T}^{2}-\omega_{L}^{2}\right)},
$$

$\omega_{T}$ - циклическая частота поперечных оптических колебаний, $e^{*}-$ эффективный заряд иона [18]:

$$
e^{*}=\kappa_{\infty} \omega_{L} \sqrt{\frac{\bar{M} a^{3}}{4 \pi \bar{\kappa}}} .
$$

Используя (1) для нахождения величины электрического поля и (2П) находим выражения для компонент вектоpa $\mathbf{r}$ лежащей в плоскости квантовой ямы и $z$-компоненты

$$
\begin{aligned}
\left(\mathbf{r}_{\|}(\boldsymbol{\rho}, z)=\right. & -i \mathbf{q}\left(b_{\mathbf{q}} \exp (i \mathbf{q r}-i \omega t)\right. \\
& \left.-b_{\mathbf{q}}^{*} \exp (-i \mathbf{q r}+i \omega t)\right) \theta\left(d_{Q W} / 2-|z|\right) \\
& \times \begin{cases}\cos \left(\pi n z / d_{Q W}\right), & n=1,3 \ldots \\
\sin \left(\pi n z / d_{Q W}\right), & n=2,4 \ldots\end{cases} \\
r_{z}(\boldsymbol{\rho}, z)= & \frac{\pi n}{d_{Q W}}\left(b_{\mathbf{q}} \exp (i \mathbf{q r}-i \omega t)\right. \\
& \left.+b_{\mathbf{q}}^{*} \exp (-i \mathbf{q r}+i \omega t)\right) \theta\left(d_{Q W} / 2-|z|\right) \\
& \times \begin{cases}-\sin \left(\pi n z / d_{Q W}\right), & n=1,3 \ldots \\
\cos \left(\pi n z / d_{Q W}\right), & n=2,4 \ldots\end{cases}
\end{aligned}
$$

где

$$
b_{\mathbf{q}}=-\frac{a_{\mathbf{q}}}{\omega_{L}} \sqrt{\frac{a^{3} \bar{\kappa}}{4 \pi \bar{M}}}
$$

Используя (4П) и (5П), из (2П) получаем выражение для полной энергии колебаний с различными q:

$$
\frac{\bar{M}}{a^{3}} \omega_{L}^{2} S d_{Q W} \sum_{\mathbf{q}} b_{\mathbf{q}} b_{\mathbf{q}}^{*}\left(q^{2}+\left(\frac{\pi n}{d_{Q W}}\right)^{2}\right) .
$$


Введем канонические переменные поля:

$$
Q_{\mathbf{q}}=\sqrt{A}\left(b_{\mathbf{q}}+b_{\mathbf{q}}^{*}\right), \quad P_{\mathbf{q}}=-i \omega \sqrt{A}\left(b_{\mathbf{q}}-b_{\mathbf{q}}^{*}\right),
$$

где

$$
A=\frac{1}{2} \frac{\bar{M}}{\Omega} S d_{Q W}\left(q^{2}+\left(\frac{\pi n}{d_{Q W}}\right)^{2}\right) .
$$

В этом случае (7П) принимает вид: $\frac{1}{2} \sum_{q} P_{\mathbf{q}}^{2}+\omega_{L}^{2} Q_{\mathbf{q}}^{2}$. Следуя стандартной процедуре, введем операторы рождения и уничтожения фонона:

$$
c_{\mathbf{q}}^{+}=\sqrt{\frac{\omega}{2 \hbar}}\left(Q_{\mathbf{q}}-\frac{i P_{\mathbf{q}}}{\omega}\right), c_{\mathbf{q}}=\sqrt{\frac{\omega}{2 \hbar}}\left(Q_{\mathbf{q}}+\frac{i P_{\mathbf{q}}}{\omega}\right) .
$$

Используя (1), (6П), (8П) и (9П) получаем выражение для оператора (2).

\section{Финансирование работы}

Исследование выполнено при поддержке совместного проекта РФФИ (грант РФФИ-ННИО_а № 21-5212020) и Deutsche Forschungsgemeinschaft (DFG Project 448961446).

\section{Конфликт интересов}

Авторы заявляют, что у них нет конфликта интересов.

\section{Список литературы}

[1] K.S. Novoselov, A.K. Geim, S.V. Morozov, D. Jiang, M.I. Katsnelson, I.V. Grigorieva, S.V. Dubonos, A.A. Firsov. Nature, 438, 197 (2005).

[2] D. Yadav, G. Tamamushi, T. Watanabe, J. Mitsushio, Y. Tobah, K. Sugawara, A.A. Dubinov, A. Satou, M. Ryzhii, V. Ryzhii, T. Otsuji. Nanophotonics 7, 741 (2018).

[3] S. Boubanga-Tombet, W. Knap, D. Yadav, A. Satou, D.B. But, V.V. Popov, I.V. Gorbenko, V. Kachorovskii, T. Otsuji. Phys. Rev. X, 10, 031004 (2020).

[4] L. Vicarelli, M.S. Vitiello, D. Coquillat, A. Lombardo, A.C. Ferrari, W. Knap, M. Polini, V. Pellegrini, A. Tredicucci. Nature Mater. 11, 865 (2012).

[5] X. Cai, A.B. Sushkov, R.J. Suess, M. M. Jadidi, G.S. Jenkins, L.O. Nyakiti, R.L. Myers-Ward, S. Li, J. Yan, D.K. Gaskill, T.E. Murphy, H.D. Drew, M. S. Fuhrer. Nature Nanotechnology 9, 814 (2014).

[6] D.A. Bandurin, D. Svintsov, I. Gayduchenko, S.G. Xu, A. Principi, M. Moskotin, I. Tretyakov, D. Yagodkin, S. Zhukov, T. Taniguchi, K. Watanabe, I.V. Grigorieva, M. Polini, G.N. Goltsman, A.K. Geim G. Fedorov. Nature Commun. 9, 5392 (2018).

[7] B. Buttner, C.X. Liu, G. Tkachov, E.G. Novik, C. Brune, H. Buhmann, E.M. Hankiewicz, P. Recher, B. Trauzettel, S.C. Zhang, L.W. Molenkamp. Nature Phys. 7, 418 (2011).

[8] S. Dvoretsky, N. Mikhailov, Yu. Sidorov, V. Shvets, S. Danilov, B. Wittman. J. Electron. Mater. 39, 918 (2010).

[9] P. Huang, E. Riccardi, S. Messelot, H. Graef, F. Valmorra, J. Tignon, T. Taniguchi, K. Watanabe, S. Dhillon, B. Plaçais, R. Ferreira, J. Mangeney. Nature Commun. 11, 863 (2020).
[10] F. Rana. Phys. Rev. B 76, 155431 (2007).

[11] F. Rana, P.A. George, J.H. Strait, J. Dawlaty, S. Shivaraman, M. Chandrashekhar, M.G. Spencer. Phys. Rev. B 79, 115447 (2009).

[12] F. Rana, J.H. Strait, H. Wang, C. Manolatou. Phys. Rev. B, 84, 045437 (2011).

[13] G. Alymov, V. Vyurkov, V. Ryzhii, A. Satou, D. Svintsov. Phys. Rev. B, 97, 205411 (2018).

[14] M.S. Zholudev, A.V. Ikonnikov, F. Teppe, M. Orlita, K.V. Maremyanin, K.E. Spirin, V.I. Gavrilenko, W. Knap, S.A. Dvoretskiy, N.N. Mihailov. Nanoscale Res. Lett. 7, 534 (2012).

[15] S.A. Tarasenko, M.V. Durnev, M.O. Nestoklon, E.L. Ivchenko, J.-W. Luo, A. Zunger. Phys. Rev. B 91, 081302(R) (2015).

[16] K. Huang, B. Zhu. Phys. Rev. B 38, 13377 (1988).

[17] V.Ya. Aleshkin, G. Alymov, A.A. Dubinov, V.I. Gavrilenko, F. Teppe. J. Phys. Commun. 4, 115012 (2020).

[18] П. Ю, М. Кардона. Основы физики полупроводников. Физматлит, М. (2002).

[19] V.Ya. Aleshkin, A.A. Dubinov, V.I. Gavrilenko, F. Teppe. J. Opt., 23, 115001 (2021).

[20] Н.Л. Баженов, К.Д. Мынбаев, Г.Г. Зегря. ФТП 49, 1206 (2015).

[21] V.Ya. Aleshkin, A.A. Dubinov, V.V. Rumyantsev, M.A. Fadeev, O.L. Domnina, N.N. Mikhailov, S.A. Dvoretsky, F. Teppe, V.I. Gavrilenko, S.V. Morozov. J. Phys.: Condens. Matter 30, 495301 (2018).

Редактор Т.Н. Василевская 